\title{
Supernova remnant evolution in uniform and non-uniform media
}

\author{
S. E. S. Ferreira ${ }^{1,2}$ and O. C. de Jager ${ }^{1}$ \\ 1 Unit for Space Physics, School of Physics, North-West University, 2520 Potchefstroom, South Africa \\ e-mail: Stefan.Ferreira@nwu.ac.za \\ 2 Centre for High Performance Computing (CHPC), CSIR Campus, 15 Lower Hope St. Rosebank, Cape Town, South Africa
}

Received 9 May 2007 / Accepted 25 October 2007

ABSTRACT

\begin{abstract}
Aims. In this work numerical simulations showing the time evolution of supernova remnants (SNRs) in uniform and non-uniform interstellar medium (ISM) are presented.

Methods. We use a hydrodynamic model including a kinematic calculation of the interstellar magnetic field. Important parameters influencing SNR evolution include the ejecta mass and energy of the remnant, as well as the ISM density and adiabatic index. Results. By varying these parameters we constructed an analytical expression giving the return time of the SNR reverse shock to the origin, in terms of these parameters. We also found that the reverse shock spends half of its time moving outward and the other half returning to the origin. Also computed is SNR evolution in non-uniform media where the blast wave moves from one medium into either a less or more dense medium. As the SNR moves into a medium of higher density a reflection wave is created at the interface between the two media which is driven back toward the center. This drives mass via a nonspherical flow away from the discontinuity. As this wave moves inward it also drags some of the ISM field lines (if the field is parallel with the interface) with it and heats the inside of the SNR resulting in larger temperatures in this region. When a SNR explodes in a medium with a high density and the blast wave propagates into a medium with a lower density, a cavity is being blown away changing the geometry of the high density region. Also, once the forward shock moves into the medium of less density a second reverse shock will start to evolve in this region.
\end{abstract}

Key words. stars: supernovae: general - ISM: supernovae remnants - methods: numerical, magnetohydrodynamics (MHD) - shock waves - ISM: cosmic rays

\section{Introduction}

A supernova explosion of a massive star will result in an expanding supernova remnant (SNR) with speed $v$ typically in the range $10^{8}$ to $10^{9} \mathrm{~cm} \mathrm{~s}^{-1}$, depending on both the ejection energy $E_{\text {ej }}$ and ejecta mass $M_{\text {ej }}$. The expansion of a SNR is decelerated due to mass-loading by the swept-up interstellar medium (ISM). During the initial stages, the ejecta mass is larger than the sweptup mass and the SNR is in the free expansion phase. See Woltjer (1972) for a description of the different stages of SNR evolution. When the swept-up mass becomes sufficiently larger than the ejecta mass (1.61 larger McKee \& Truelove 1995) the SNR is in the Sedov (Sedov 1959) phase. During this phase a reverse shock (RS) will form because of the low pressure of the ejected material which have been adiabatically expanding. The RS will then be driven back into the interior of the SNR (e.g. Ardavan 1973; McKee 1974), and in the process heat the material inside. The remnant is still bounded by a strong blast wave, called the forward shock (FS) and for this phase the FS radius increases as $t^{2 / 5}$.

In this work we begin by showing how different parameters, such as $E_{\mathrm{ej}}, M_{\mathrm{ej}}$, ISM density $\rho_{\mathrm{ISM}}$ (see also e.g. Dwarkadas \& Chevalier 1998; Truelove \& McKee 1999; Tang \& Wang 2005) and the adiabatic index $\gamma$ (e.g. Decourchelle et al. 2000; Ellison et al. 2004) may influence the evolution of a SNR in an uniform medium. We derive an analytical expression giving the return time $t_{\mathrm{R}}$ of the RS to the origin in terms of these parameters. For this we use a hydrodynamic model (solving the well known Euler equations). Different from previous studies, we also include a kinematic calculation (solving the induction equation) of the interstellar magnetic field (LeVeque 2002; Fahr et al. 2000;
Scherer \& Ferreira 2005; Ferreira \& Scherer 2006). We also neglect the existence of a pulsar wind nebulae (PWN) inside a SNR in our calculations. As shown by van der Swaluw et al. (2001); Blondin et al. (2001); Bucciantini et al. (2003); Del Zanna et al. (2004) this relativistic wind may alter $t_{\mathrm{R}}$ making our calculations only valid for SNR without any PWN or with a PWN but having a relatively small luminosity. Also not included are the contribution from a late phase stellar wind. This, in particular might be important for a high mass progenitor. The effect of such a circumstellar wind and how this may modify conditions in the ambient medium is a topic of future research.

For the second part we calculate SNR evolution in nonuniform media (see also Voelk 1983; Tenorio-Tagle et al. 1991; Borkowski et al. 1997; Dohm-Palmer \& Jones 1996; Jun \& Jones 1999). We concentrate on the simple scenario where a SNR moves from one medium into either a less or more dense medium. For simplicity we assume that the interface between these two media is an infinite plane although simulations of more complex geometries are possible (e.g. incursions in the plane, relative motion etc.). It is shown how different parameters, such as density, speed, pressure and the compressed ISM magnetic field changes when a SNR moves from one into another medium with a different density (e.g. from the ISM into a molecular cloud or vice versa).

\section{Model and parameters}

To model the evolution of a SNR the Euler equations

$\frac{\partial}{\partial t} \rho+\nabla \cdot(\rho \boldsymbol{v})=0$ 
$\frac{\partial}{\partial t}(\rho \boldsymbol{v})+\nabla \cdot(\rho \boldsymbol{v} \boldsymbol{v}+P \boldsymbol{I})=0$,

$\frac{\partial}{\partial t}\left(\frac{\rho}{2} \boldsymbol{v}^{2}+\frac{P}{\gamma-1}\right)+\nabla \cdot\left(\frac{\rho}{2} \boldsymbol{v}^{2} \boldsymbol{v}+\frac{\gamma \boldsymbol{v} P}{\gamma-1}\right)=0$

describing inviscid flow are solved with $\rho$ the density, $v$ the velocity and $P$ the gas pressure. These equations correspond to the Navier-Stokes equations with zero viscosity and no heat conduction. They describe the balance of mass, momentum and energy. Currently we only consider a one fluid scenario with non-relativistic speeds, e.g. an adiabatic index of $5 / 3$. The numerical scheme is discussed in LeVeque (2002) and compute solutions to hyperbolic differential equations using a wave propagation approach. Following is a short summary of the scheme from LeVeque (2002):

Consider a homogeneous conservation law

$\frac{\partial q}{\partial t}+\frac{\partial f(q)}{\partial x}$

with flux function $f(q)$. For a homogeneous system, a Riemann solver is used that for any two states $Q_{i-1}$ and $Q_{i}$ returns a set of $M_{w}$ waves $W_{i-1 / 2}^{p}$ and speeds $s_{i-1 / 2}^{p}$ satisfying

$\sum_{p=1}^{M_{w}} W_{i-1 / 2}^{p}=Q_{i}-Q_{i-1} \equiv \triangle Q_{i-1 / 2}$.

The solver also returns a left-going fluctuation $A^{-} \triangle Q_{i-1 / 2}$ and a right-going fluctuation $A^{+} \triangle Q_{i-1 / 2}$ which should satisfy

$A^{-} \triangle Q_{i-1 / 2}+A^{+} \triangle Q_{i-1 / 2}=f\left(Q_{i}\right)-f\left(Q_{i-1}\right)$

and where e.g.

$A^{-} \Delta Q_{i-1 / 2}=\sum_{p}\left(s_{i-1 / 2}^{p}\right)^{-} W_{i-1 / 2}^{p}$

with $s^{-}=\min (s, 0)$ and $s^{+}=\max (s, 0)$. The Riemann solver first computes waves and speeds and then uses these to compute $A^{-} \triangle Q_{i-1 / 2}$ and $A^{+} \triangle Q_{i-1 / 2}$. These are then used in a high resolution method which takes the form

$$
\begin{array}{r}
Q_{i}^{n+1}=Q_{i}^{n}-\frac{\Delta t}{\Delta x}\left[A^{+} \Delta Q_{i-1 / 2}+A^{-} \Delta Q_{i-1 / 2}\right] \\
-\frac{\Delta t}{\Delta x}\left(F_{i+1 / 2}-F_{i-1 / 2}\right)
\end{array}
$$

where

$F_{i-1 / 2}=\frac{1}{2} \sum_{p=1}^{M_{w}}\left|s_{i-1 / 2}^{p}\right|\left(1-\frac{\Delta t}{\Delta x}\left|s_{i-1 / 2}^{p}\right|\right) W_{i-1 / 2}^{p}$

where $W_{i-1 / 2}^{p}$ now represents a limited version (either superbee or Van Leer) of the original wave obtained by e.g. comparing $W_{i-1 / 2}^{p}$ to $W_{i-3 / 2}^{p}$ if $s^{p}>0$. See also Fahr et al. (2000), Scherer \& Ferreira (2005), Ferreira \& Scherer (2006) for an application of this model to calculate the heliosphere.

To compute the compressed ISM magnetic field we solve

$$
\frac{\partial \boldsymbol{B}}{\partial t}+\nabla \times(\boldsymbol{v} \times \boldsymbol{B})=0
$$

using a similar scheme as for the fluid part. Note that this is not a full MHD solution because the field is calculated kinematically from the flow (Scherer \& Ferreira 2005; Ferreira \& Scherer 2006) and no backreaction on the fluid is considered. In this work we only consider the field of the ISM which gets compressed as the ISM is swept-up by the forward shock of the SNR. The model solves these equations in spherical coordinates $r$ and $\phi$, with $r$ ranging from $0.1 \mathrm{pc}$ to $10-20 \mathrm{pc}$ (1000 gridpoints) and $\phi$ from $0^{\circ}$ to $180^{\circ}$ (150 gridpoints).

For the initial and boundary conditions (see also Blondin \& Ellison 2001; van der Swaluw et al. 2001; Bucciantini et al. 2003; Del Zanna et al. 2004) we assume a spherical region, radius $r_{\mathrm{ej}}$, and a high constant density $\rho_{\mathrm{ej}}$ with a radially increasing velocity profile

$v=\frac{r}{t}=v_{\mathrm{ej}} r / r_{\mathrm{ej}}$.

In this case we take $r_{\mathrm{ej}}=0.1 \mathrm{pc}$ while for the density we have

$\rho_{\mathrm{ej}}=\frac{3 M_{\mathrm{ej}}}{4 \pi r_{\mathrm{ej}}^{3}}$

with $M_{\mathrm{ej}}$ the ejecta mass. For the velocity we have

$v_{\mathrm{ej}}=\sqrt{\frac{10}{3} \frac{E_{\mathrm{ej}}}{M_{\mathrm{ej}}}}$.

Figure 1 shows a typical example of a SNR at different stages of evolution computed using $E_{\mathrm{ej}}=10^{51} \mathrm{erg}, M_{\mathrm{ej}}=3 M_{\odot}=$ $6 \times 10^{33} \mathrm{~g}$ and for the background ISM we have $\rho_{\text {ISM }}=0.9 \times$ $10^{-24} \mathrm{~g} \mathrm{~cm}^{-3}$ (see also van der Swaluw et al. 2001). Shown in the top panel is the ISM magnetic field $B$ (assumed to be perpendicular to the flow) which gets compressed by the FS by a factor $s$. From the Rankine-Hugoniot conditions one can show that for non-relativistic speeds we have $\gamma=5 / 3$ and $s \approx 4$ which is the factor shown in Fig. 1.

Shown in the second panel from the top is the density $\rho$. From the origin one can see the RS, the contact discontinuity between the ejected and shocked ISM and the FS propagating in the undisturbed ISM. Shown here is that for these parameters the swept-up material is comparable to the ejected mass around $1000 \mathrm{yr}$. As time increases the swept up mass becomes considerably larger than the ejecta mass and the SNR enters the Sedov phase where the FS radius now scale as $t^{2 / 5}$. Note that, depending on parameters, the transition between these phases maybe just as long as the phase itsel (see e.g. Cioffi et al 1988; Dohm-Palmer \& Jones 1996).

In the bottom three panels the speed $v$ and pressure $P$ are shown. Note that because $v$ is shown on a logarithmic scale we show $-v$ below. This is important to show when the flow changes direction and points inward. Shown in these three panels is that at some time the RS starts to move inward because of the low pressure of the ejected material which has been adiabatically expanding. At this stage the speed reverses sign meaning that the flow is now toward the center. As shown for $4000 \mathrm{yr}$ the RS have reached the origin. As shown in the bottom panel when the $\mathrm{RS}$ propagates into the ejecta the latter gets heated. In the next section we will concentrate on deriving an analytical expression giving the return time of the RS to the origin in terms of different parameters.

\section{SNR evolution in an uniform medium}

In this section a parameter study is done to investigate the effects of different parameters on SNR evolution. In particular the time for the RS to return to the origin $t_{\mathrm{R}}$ is investigated. Note that although the rotational energy released by a pulsar (which may be found inside a SNR) is much smaller compared to the mechanical energy released in the SNR the possible existence of a 


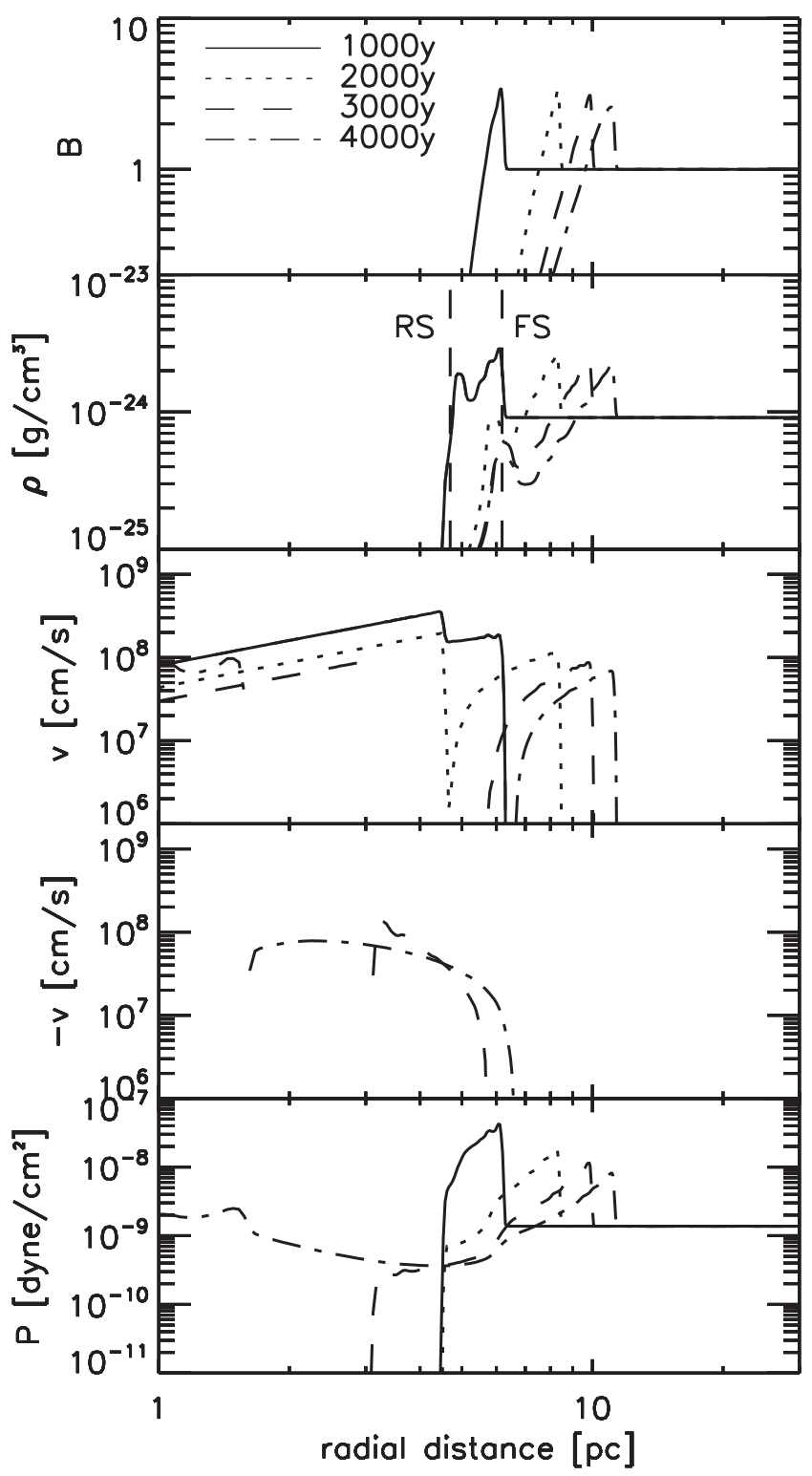

Fig. 1. The evolution of a typical SNR computed using parameters as described in the text. Results are shown from 1000 to $4000 \mathrm{yr}$ as indicated. Shown in the top panel is the ISM magnetic field $B$ (perpendicular to the flow) which gets compressed by a factor $s \approx 4$. Shown in the second panel is the density $\rho$. From the origin one can see the reverse shock (RS), the contact discontinuity between the ejected and shocked ISM and the blast wave, forward shock (FS), which propagates in the undisturbed ISM. The position of the RS and FS are indicated by the vertical dashed lines. In the bottom panels the speed $v$ is shown on a logarithmic scale, and therefore $-v$ is shown just below it. Shown here is that at some time the RS starts to move inward, and the speed reverses sign meaning that the flow is now inward toward the center. In the last panel the pressure $P$ is shown.

relativistic wind, PWN, inside a SNR may alter the return time of the RS to the origin (van der Swaluw et al. 2001; Blondin et al. 2001; Bucciantini et al. 2003; Del Zanna et al. 2004). Initially the pressure in the pulsar bubble is negligible compared to the SNR and the PWN has no effect on the dynamics of the infalling reverse shock (e.g. Blondin et al. 2001). However, as the pulsar bubble is compressed the pressure rises and at some stage becomes comparable to the pressure behind the RS leading to an unstable phase.
In this work we neglect the effect of such a PWN making our expression of $t_{\mathrm{R}}$ valid for SNRs with no PWN inside, or where the luminosity of the PWN is relatively small. The effect of the PWN on the SNR dynamics is expected to be negligible if the total energy imparted to the FS during the contact time, $T_{p}$, between this shock and the relativistically expanding PWN during the early phases of evolution is much less than the blast energy. This is if

$I \Omega_{0} \dot{\Omega}_{0} T_{p} \ll E_{\mathrm{SN}}$

where $I \Omega_{0} \dot{\Omega}_{0}$ is the spindown power at birth. Here we have assumed that the contact time $T_{p}$ is less than the pulsar spindown timescale $\tau=\frac{1}{2} I \Omega_{0}^{2} / I \Omega_{0} \dot{\Omega}_{0}$. If the latter condition is not met (e.g. $\tau<T_{p}$ ) the condition for the FS not to be modified by the PWN becomes

$\frac{1}{2} I \Omega_{0}^{2} \ll E_{\mathrm{SN}}$

so that we compare the total kinetic energy with the blast energy. The range of relevant above mentioned parameters and those influencing the reverse shock return time at later stages will be investigated in a seperate paper.

Figure 2 shows the computed FS (top panel) and RS (bottom panel) radius of such a SNR as a function of time. Shown are different scenarios corresponding to a SNR expanding in ISM of different densities, e.g. $\rho_{\text {ISM }}=10^{-27} \mathrm{~g} \mathrm{~cm}^{-3}$ up to $\rho_{\text {ISM }}=$ $10^{-23} \mathrm{~g} \mathrm{~cm}^{-3}$. Shown on the left panels are SNR corresponding to an ejecta mass $M_{\mathrm{ej}}=3 M_{\odot}$ in the middle panels $M_{\mathrm{ej}}=2 M_{\odot}$ and on the right $M_{\mathrm{ej}}=3 M_{\odot}$. The dotted lines in the upper left panel show the Sedov solution where the FS radius increases as $t^{2 / 5}$.

As expected, the FS radius is largely dependent on the ISM density. Larger densities, e.g. $\rho_{\text {ISM }}=10^{-23} \mathrm{~g} \mathrm{~cm}^{-3}$ leads to a much smaller blast wave radius compared to lesser densities, e.g. $\rho_{\text {ISM }}=10^{-27} \mathrm{~g} \mathrm{~cm}^{-3}$ for the same simulation time. Also the ejecta mass $M_{\mathrm{ej}}$ does not play an significant role in determining the FS radius, especially for the times shown toward the end of the simulation. This is because after a few hundred years the mass of the swept-up material is comparable or higher than the ejecta mass. This is not the case for first few hundred years where this quantity will result in a different initial speed (via Eq. (13)) and may influence the transition time from a free expansion to the adiabatic phase.

The bottom panels in Fig. 2 show that the RS radius and also the time for it to return to the origin is very much dependent on both $\rho_{\text {ISM }}$ and $M_{\text {ej }}$. For a larger $\rho_{\text {ISM }}$ the SNR evolves sooner into the Sedov phase (by comparison of the computed FS radius in the upper left panel to the dotted lines). This is also the case for a smaller $M_{\mathrm{ej}}$ which will lead to a smaller pressure of the ejecta material. Examining Fig. 2 shows that the density of the ISM is an important parameter determining the RS radius and return time because this parameter may vary many orders of magnitude.

The RS return time $t_{\mathrm{R}}$ for the different parameters shown in Fig. 2 can be summarized in the left panel in Fig. 3. Shown here is $t_{\mathrm{R}}$ as a function of $\rho_{\mathrm{ISM}}$ for different $M_{\mathrm{ej}}$. As shown for $M_{\mathrm{ej}}=1,2,3,5,7$ and $10 M_{\odot}, t_{\mathrm{R}} \propto \rho_{\mathrm{ISM}}^{-1 / 3}$, similar to the result from Truelove \& McKee (1999). Shown on the top right panel is the dependence of $t_{\mathrm{R}}$ on $M_{\mathrm{ej}}$ for all different $\rho_{\mathrm{ISM}}$ scenarios. As shown here, $t_{\mathrm{R}} \propto M_{\mathrm{ej}}^{3 / 4}$ for all $\rho_{\text {ISM }}$ scenarios.

The middle right panel shows the dependence of $t_{\mathrm{R}}$ to the ejection energy $E_{\mathrm{ej}}$. Computations were done by assuming $E_{\mathrm{ej}}=$ $0.5 \times 10^{51}, 1 \times 10^{51}, \ldots, 10 \times 10^{51}$ erg respectively. Shown 

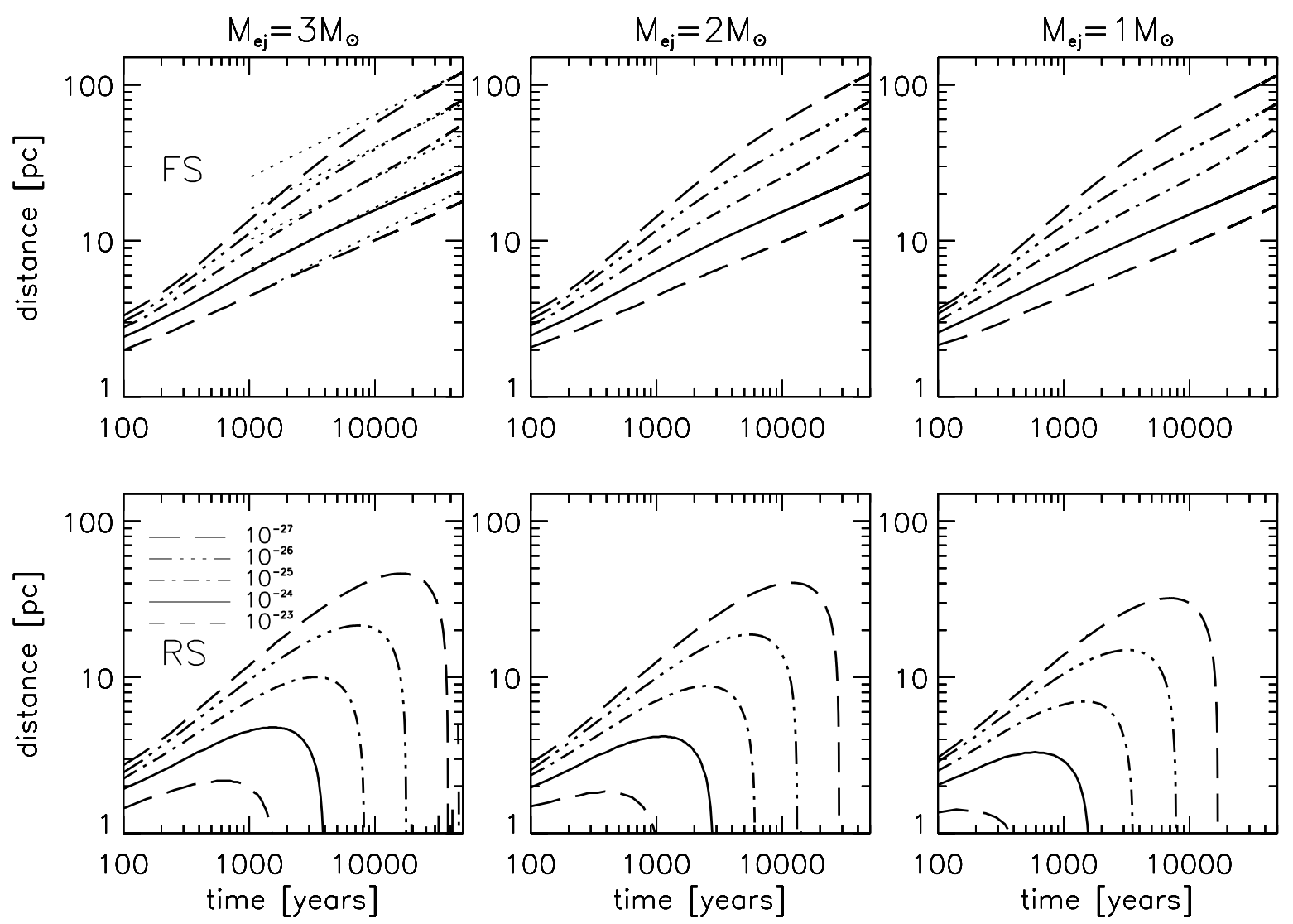

Fig. 2. The computed FS (top panel) and RS (bottom panel) radius as a function of time. Different scenarios are shown corresponding to a SNR expanding in ISM of different densities, e.g. $\rho_{\mathrm{ISM}}=10^{-27} \mathrm{~g} \mathrm{~cm}^{-3}$ up to $\rho_{\mathrm{ISM}}=10^{-23} \mathrm{~g} \mathrm{~cm}^{-3}$. Shown on the left panels are SNR scenarios corresponding to an ejecta mass $M_{\mathrm{ej}}=3 M_{\odot}$ in the middle panels $M_{\mathrm{ej}}=2 M_{\odot}$ and on the right $M_{\mathrm{ej}}=1 M_{\odot}$. The dotted lines in the upper left panel show the Sedov solution where the FS radius increases as $t^{2 / 5}$.

here is that when the return time $t_{\mathrm{R}}$ of the RS is calculated, $t_{\mathrm{R}} \propto E_{\mathrm{ej}}^{-45 / 100}$. Note that this dependence on mass and energy is slightly different from the similarity scaling of Truelove \& McKee (1999) which yielded -0.833 and -0.5 respectively. This might be due to the pressure of the ISM where Truelove \& McKee (1999) assumed $P=0$ while in our model the ISM temperature is assumed to be $1000 \mathrm{~K}$ (see also e.g. Tang \& Wang 2005).

Another important parameter influencing the shock structure in particular, is the adiabatic index $\gamma$. The latter may change due to the non-linear interaction of accelerated cosmic rays on SNR shocks (e.g. Decourchelle et al. 2000; Ellison et al. 2004). As these relativistic particles are produced they contribute to the pressure resulting in the shocked plasma becoming more compressible $(\gamma \rightarrow 4 / 3)$. Shown in Fig. 3 is $t_{\mathrm{R}}$ for values $\gamma=5 / 3,3 / 2$ and $4 / 3$ with the first and last values probably upper and lower limits of this parameter. As shown, $t_{\mathrm{R}} \propto \gamma^{-3 / 2}$.

Therefore concluding from Fig. 3 we can write the return time of the RS to the origin as

$t_{\mathrm{R}}=4000\left(\frac{\rho_{\mathrm{ISM}}}{\rho_{0}}\right)^{-1 / 3}\left(\frac{M_{\mathrm{ej}}}{M_{0}}\right)^{3 / 4}\left(\frac{E_{\mathrm{ej}}}{E_{0}}\right)^{-45 / 100}\left(\frac{\gamma}{\gamma_{0}}\right)^{-3 / 2} y$.

In these equations $\rho_{0}=10^{-24} \mathrm{~g} \mathrm{~cm}^{-3}, M_{0}=3 M_{\odot}, E_{0}=10^{51} \mathrm{erg}$ and $\gamma_{0}=5 / 3$. Assuming these values result in $t_{\mathrm{R}}=4000 \mathrm{yr}$.

It is interesting to note the time at which the RS start to move inward toward the origin, $t_{\mathrm{c}}$. Figure 4 shows $t_{\mathrm{c}}$ as a function of $t_{\mathrm{R}}$. Shown here are values for all our calculations shown in Fig. 3. As shown $t_{\mathrm{c}}=0.5 t_{\mathrm{R}}$ for all our computations.

\section{SNR evolution in a non-uniform medium}

In the previous section the evolution of SNRs have been computed as spherical explosions within a uniform ISM. The problem becomes more complex when the surrounding medium is not uniform (see e.g. Voelk 1983). Tenorio-Tagle et al. (1991) have done hydrodynamic simulations of SNRs interactions with spherical bubbles. Borkowski et al. (1997) have computed the collision between the ejecta of e.g. SN 1987A and its circumstellar ring. Also Dohm-Palmer \& Jones (1996) explored the dynamics of SNRs which developed on the edge of a giant molecular cloud. This was followed by Jun \& Jones (1999) computing the expansion of a SNR into an ISM containing different clouds. These studies all confirm that the evolution of SNRs is more complex in non-uniform media.

In this section SNR evolution in non-uniform media is investigated. An example of this is an SNR moving from one ISM medium to another with different densities, e.g. from the ISM into a molecular cloud. We will restrict ourselves with the simple case where the contact discontinuity is an infinite plane. Also numerical noise in the form of density perturbations are not included in our computations (e.g. Blondin \& Ellison 2001; Bucciantini et al. 2004). Instead we focus firstly on the interaction of an SNR moving into a denser medium and the subsequent phenomena which will occur. In particular we are interested in answering questions such as: What happens to the medium of enhanced density (e.g. molecular cloud) during and after the collision? What happens to the compressed interstellar magnetic field? What velocities and shocks/waves are induced due to this collision? etc. In the last part we concentrate on the 

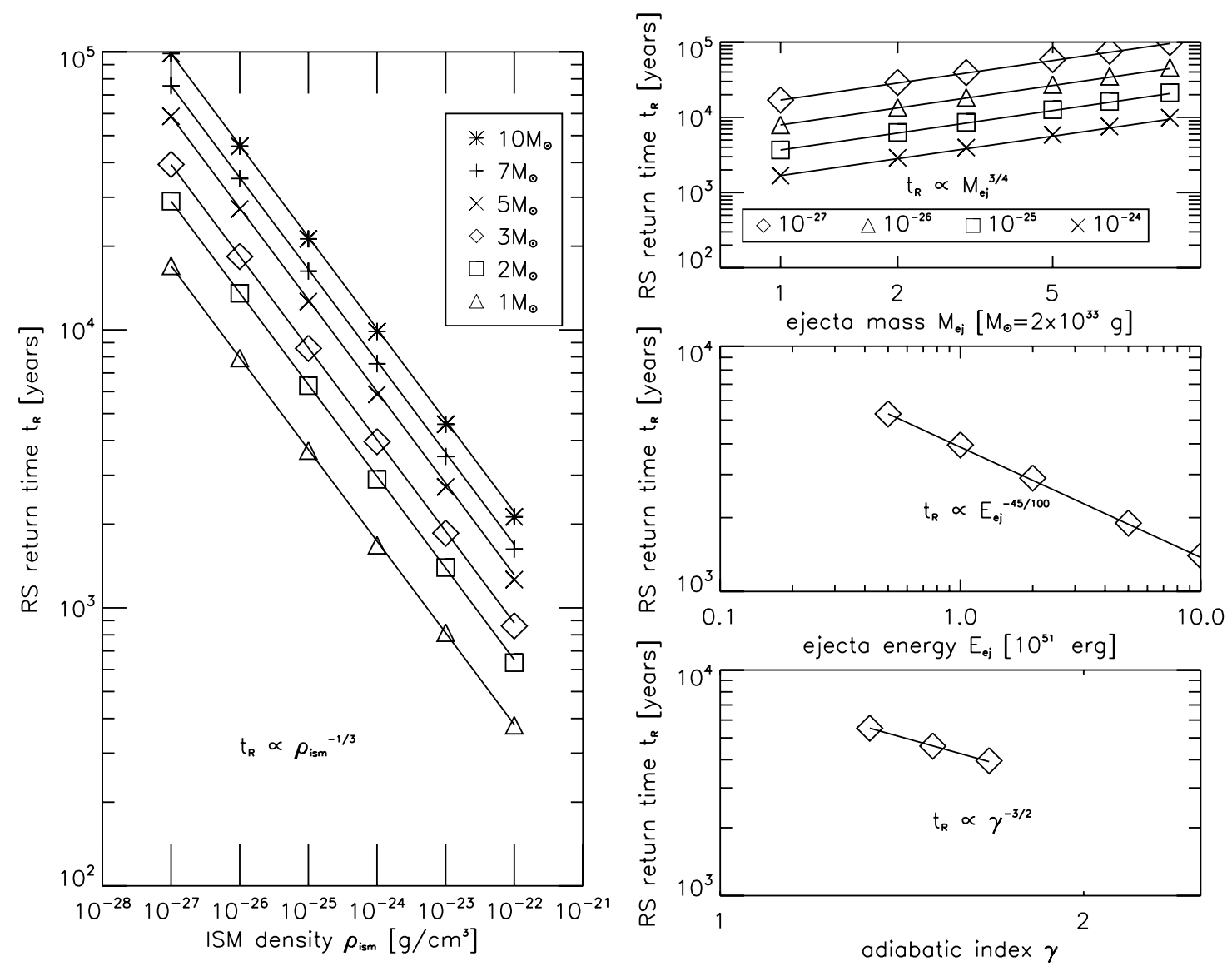

Fig. 3. The effect of different parameters on the RS return time $t_{\mathrm{R}}$. Shown in the left large panel is $t_{\mathrm{R}}$ as a function of $\rho_{\mathrm{ISM}}$ for $M_{\mathrm{ej}}=1,2,3, \ldots ., 10 M_{\odot}$. Shown on the top right panel is the dependence of $t_{\mathrm{R}}$ on $M_{\mathrm{ej}}$ for all different $\rho_{\mathrm{ISM}}$ scenarios. The middle panel on the right show the dependence of $t_{\mathrm{R}}$ on the ejection energy $E_{\mathrm{ej}}$ and the bottom right panel the dependence on the adiabatic index $\gamma$.

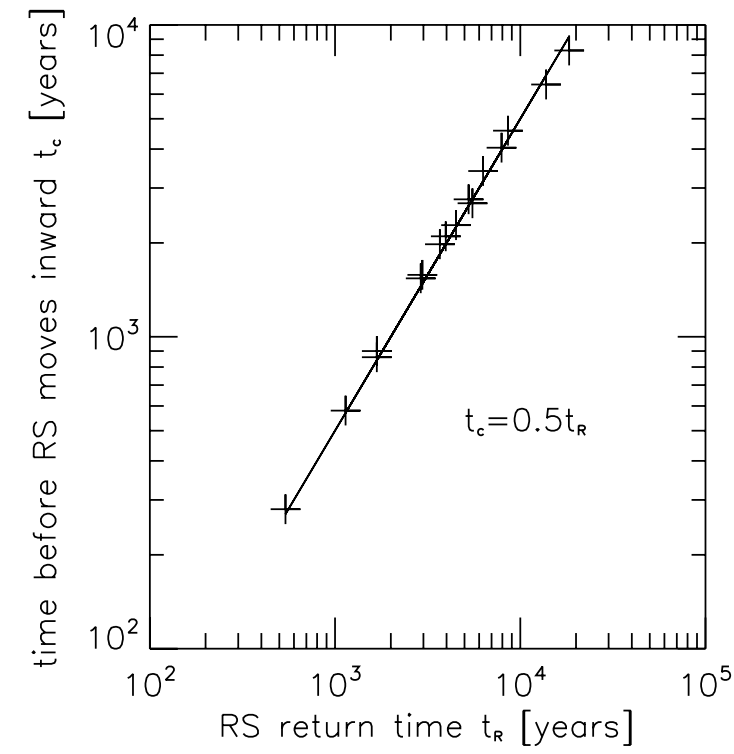

Fig. 4. Time before the RS starts to move inward toward the origin $t_{\mathrm{c}}$ as a function of $t_{\mathrm{R}}$ for all computations as shown in Fig. 2. Shown by the solid line is $t_{\mathrm{c}}=0.5 t_{\mathrm{R}}$.

opposite situation where a SNR is moving into a region of lesser density.

Figure 5 shows an example of the interaction of a typical SNR with a medium of enhanced density. Results are shown in terms of the density for an SNR moving from an ISM density of $\rho_{\text {ISM }}=10^{-24} \mathrm{~g} \mathrm{~cm}^{-3}$ to a medium where $\rho_{\text {ISM }}=10^{-23} \mathrm{~g} \mathrm{~cm}^{-3}$. The contact discontinuity is an infinite plane situated 5 pc away from the initial explosion. Shown from top to bottom are different stages in the SNR evolution, e.g. 1000, 3000, 5000 and 7000 yr.

Figure 5 shows that as the SNR moves into the higher density medium two important effects occur. Firstly, the blast wave (FS) decelerates because of the increase in mass (momentum conservation). This results in the SNR geometry deviating significantly from a sphere as time increases. Secondly, due to the pressure imbalance, a reflection wave is created which is driven back toward the center. This now drives mass via nonspherical flow from the medium of higher to the medium of lower density resulting in the denser medium no longer exhibiting its planar geometry with a large indent created by interaction with the SNR.

Figure 6 shows the compressed ISM magnetic field. For this our model was extended to 3D to calculate the full MHD equations. Shown on the left is a scenario where the ISM magnetic field is parallel and on the right when it is perpendicular to the ISM interface. As the blast wave moves outward, the field is compressed by a factor $s \sim 4$. In the case when the FS hits the medium of higher density the reflection wave immediately moves in the opposite direction dragging some of the field lines with it for the parallel case. As material gradually moves toward the center of the SNR the compressed field is transported with it. The result is that the field inside the SNR close to the higher density region is now considerably larger. For the perpendicular 


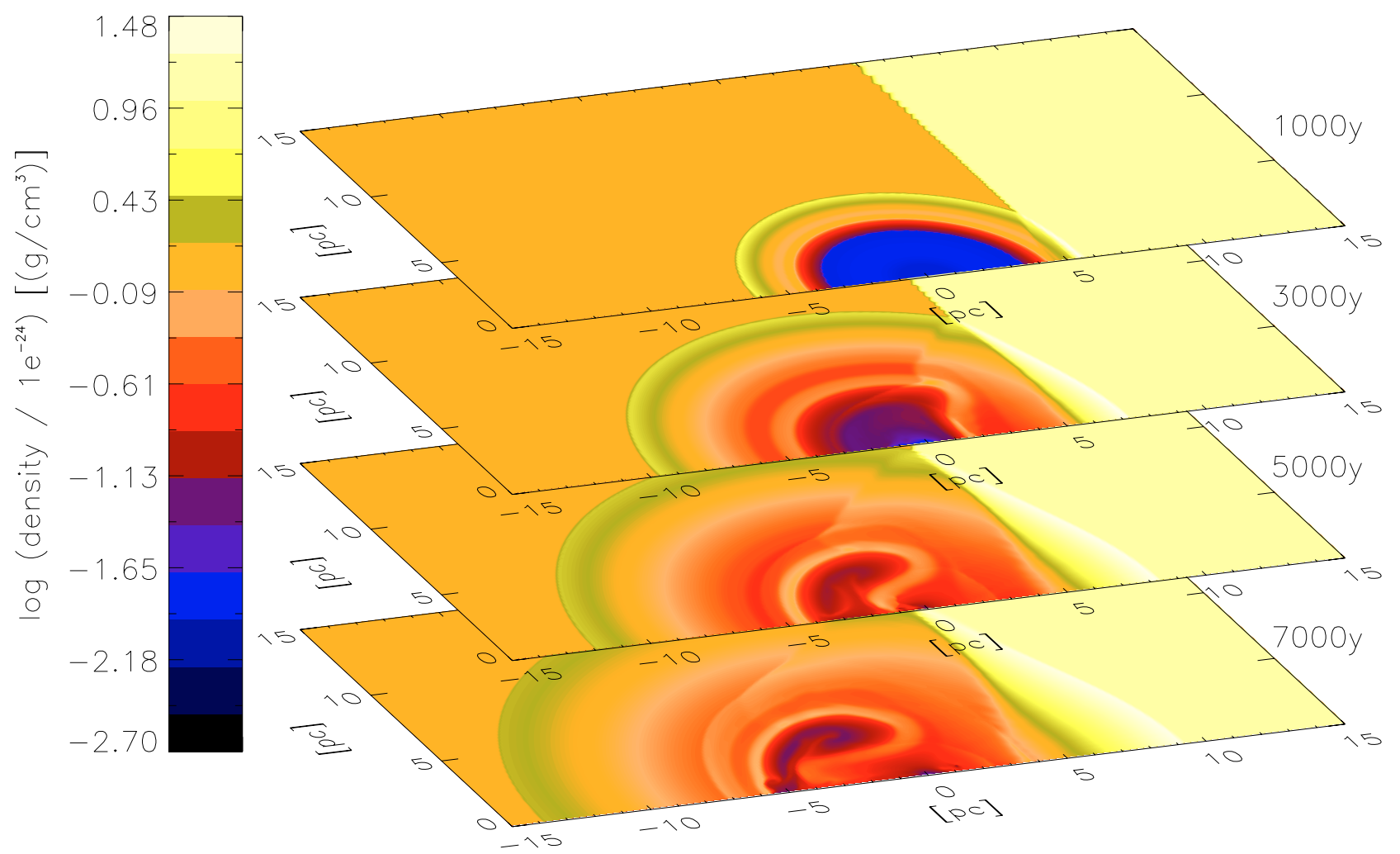

Fig. 5. A computed SNR (in terms of density) moving from a ISM density of $\rho_{\text {ISM }}=10^{-24} \mathrm{~g} \mathrm{~cm}^{-3}$ to a medium where $\rho_{\text {ISM }}=10^{-23} \mathrm{~g} \mathrm{~cm}^{-3}$. Shown from top to bottom are different stages in the SNR evolution, e.g. 1000, 3000, 5000 and 7000 yr. Note that a logarithmic scaling is used and that the density is normalized by dividing by $10^{-24}$.

case shown on the right this does not occur and the field inside remains largely unaffected by the reflection wave.

These effects can be more qualitatively shown in Fig. 7. The figure is similar as Fig. 1, except computations are shown as radial profiles from the SNR origin in the direction directly toward the more denser medium (solid line) and directly away in the opposite direction (dotted line). The left panels show the parameters at $1000 \mathrm{yr}$, the middle panel at $1500 \mathrm{yr}$ and the right panel the parameters at $2000 \mathrm{yr}$.

Most interesting in Fig. 7 is the reflected wave which can be easily identified in the bottom panel by a large gradient in pressure. The location of this wave is shown by the dashed line in each panel. Shown here is that this wave is almost immediately created as the FS reaches the denser medium and start to move inward. This is due to the pressure imbalance where the part of the remnant expanding in the more dense medium is strongly decelerated (shown by the third and fourth panels). Also of interest is that this reflected wave catches up with the RS because of the larger speed. As this wave propagates inward it heats the inside of the SNR resulting in larger temperatures compared to the opposite side of the explosion. Note that for the simulations in this section we assumed $M_{\mathrm{ej}}=1.4 M_{\odot}$ where from Eq. (16) we would expect the RS to reach the center at 2258 yr. Also evident in Fig. 7 is waves propagating back and fourth directly behind the reflected wave as it moves inward. This is not evident in the opposite direction.

Lastly, we will concentrate on a scenario where the opposite occurs. Here a SNR explodes in a medium with a relative high density and the blast wave then propagates into a medium with a lower density. Again we will assume that the contact discontinuity between the two media is in the form of a infinite plane situated 5 pc away from the initial explosion. The results are shown in Fig. 8 which is similar to Fig. 5 except here the SNR moves from a medium with a density of $\rho_{\text {ISM }}=10^{-24} \mathrm{~g} \mathrm{~cm}^{-3}$ into a medium where $\rho_{\text {ISM }}=10^{-25} \mathrm{~g} \mathrm{~cm}^{-3}$. In this case, densities of $\rho_{\text {ISM }}=10^{-24} \mathrm{~g} \mathrm{~cm}^{-3}$ will be the denser region.

As shown in Fig. 8 when the SNR moves into a less dense medium it accelerates and the geometry deviates from a sphere. Compared to the previous scenario there is no reflected wave moving inward, e.g. the the flow is always outward until the RS start to move inward. Also a supernova explosion inside a dense medium moving into a less dense medium will result in a cavity being blown away by the blast wave and may significantly change the geometry at the interface between the two media.

Figure 9 shows the ISM magnetic field which gets compressed by the FS. Again two scenarios are shown where the field is parallel (left panels) and perpendicular (right panels) to the interface. Shown here is that in contrast to the previous parallel scenario (left panel in Fig. 6) the enhanced field is not transported inwards. From a magnetic point of view, the two scenarios differ significantly. For the first scenario the enhanced field is dragged into the interior of the SNR due to the reflection at the interface. This leads to a much stronger field inside compared to the scenario shown in Fig. 9. From a diffusive shock acceleration point of view one can expect large differences in cosmic ray intensities found between these two scenarios. Concentrating on Fig. 9 the side in the higher density shows a spherical geometry while the side in the lower density shows an almost plane geometry because of the larger radius after the same simulation time. The difference in the radius of the SNR blast wave in the two different media alone can lead to different curvature cutoff effects in the accelerated cosmic ray spectrum.

Figure 10 shows the radial profiles of the different parameters. As in Fig. 7, the values in the direction directly to the interface between the media are shown as the solid line, while the 

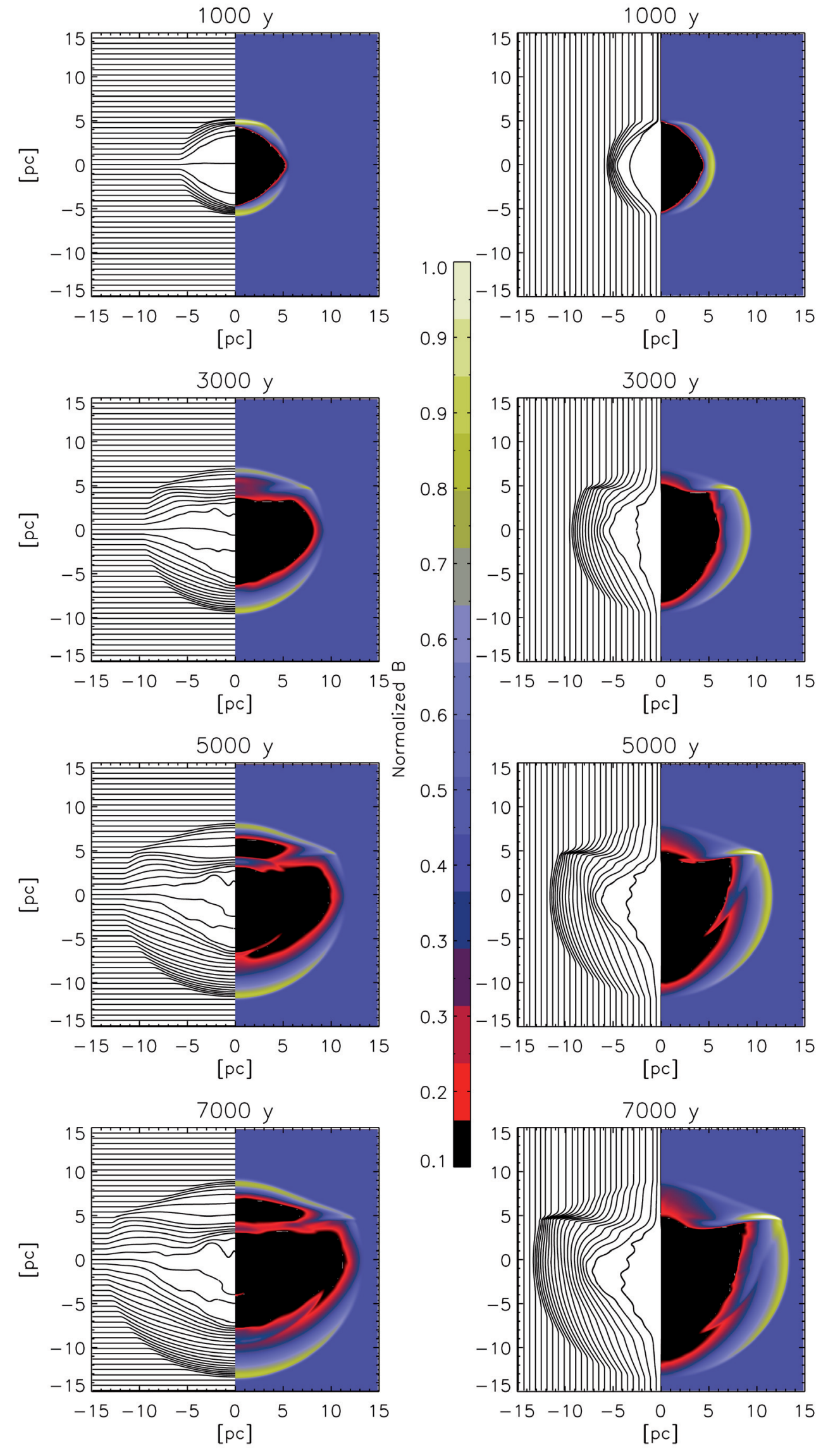

Fig. 6. As in Fig. 5 except now the compressed ISM magnetic field is shown for two different ISM field orientations. Shown on the left of each panel are the field lines and on the right the field magnitude. 
S. E. S. Ferreira and O. C. de Jager: Supernova remnant evolution in uniform and non-uniform media

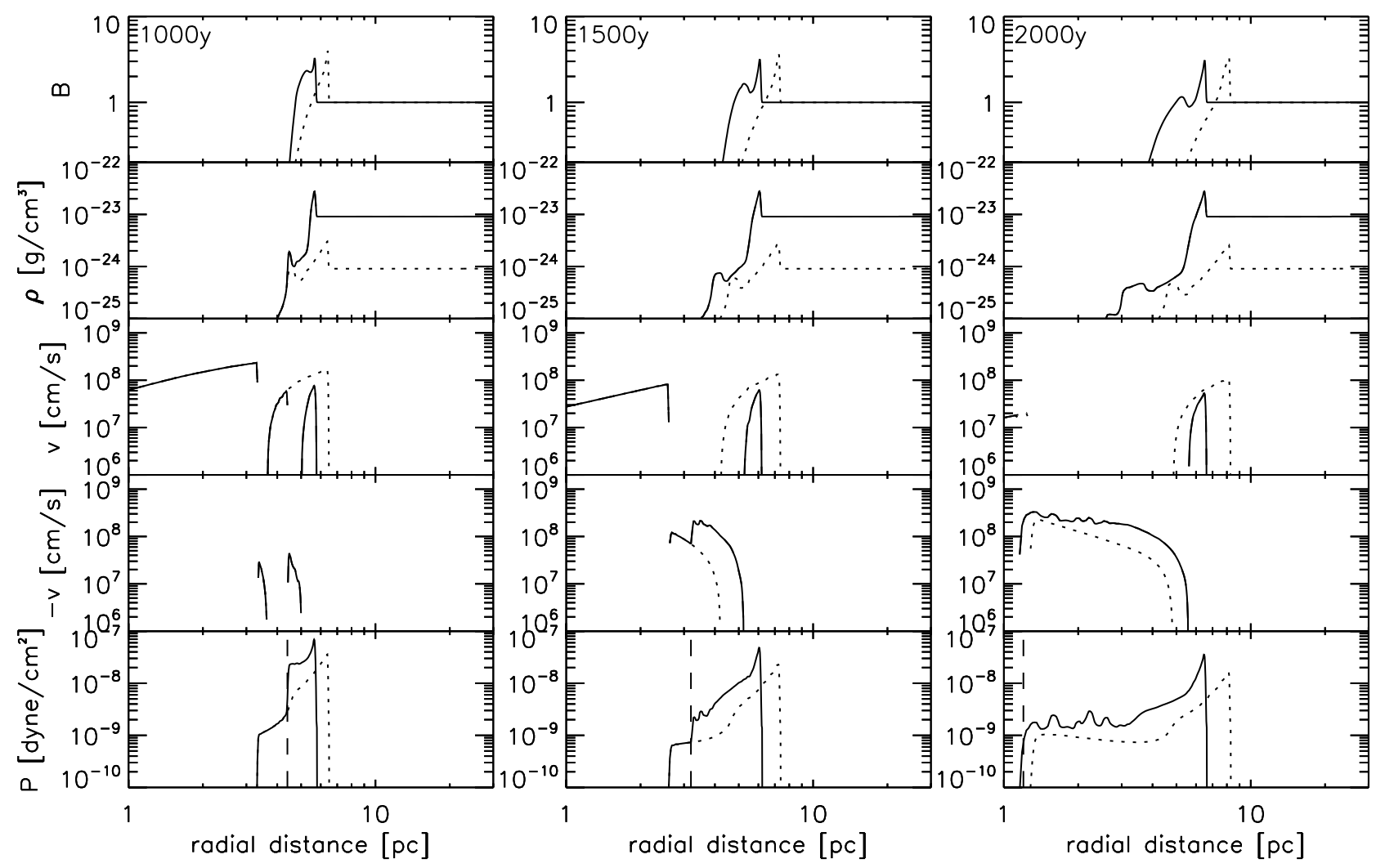

Fig. 7. The top panel shown the ISM magnetic field $B$ (for the case where the field is parallel to the interface). Shown in the second panel is the density $\rho$. The bottom three panels show the speed $v$ and $-v$ and pressure $P$. Computations are shown from the SNR origin in the direction directly toward the more denser medium (solid line) and directly away in the opposite direction (dotted line). The left panels show parameters at 1000 yr, the middle panel parameters at $1500 \mathrm{yr}$ and the right panel at $2000 \mathrm{yr}$. The location of the reflected wave is shown by the dashed line in the pressure panels.

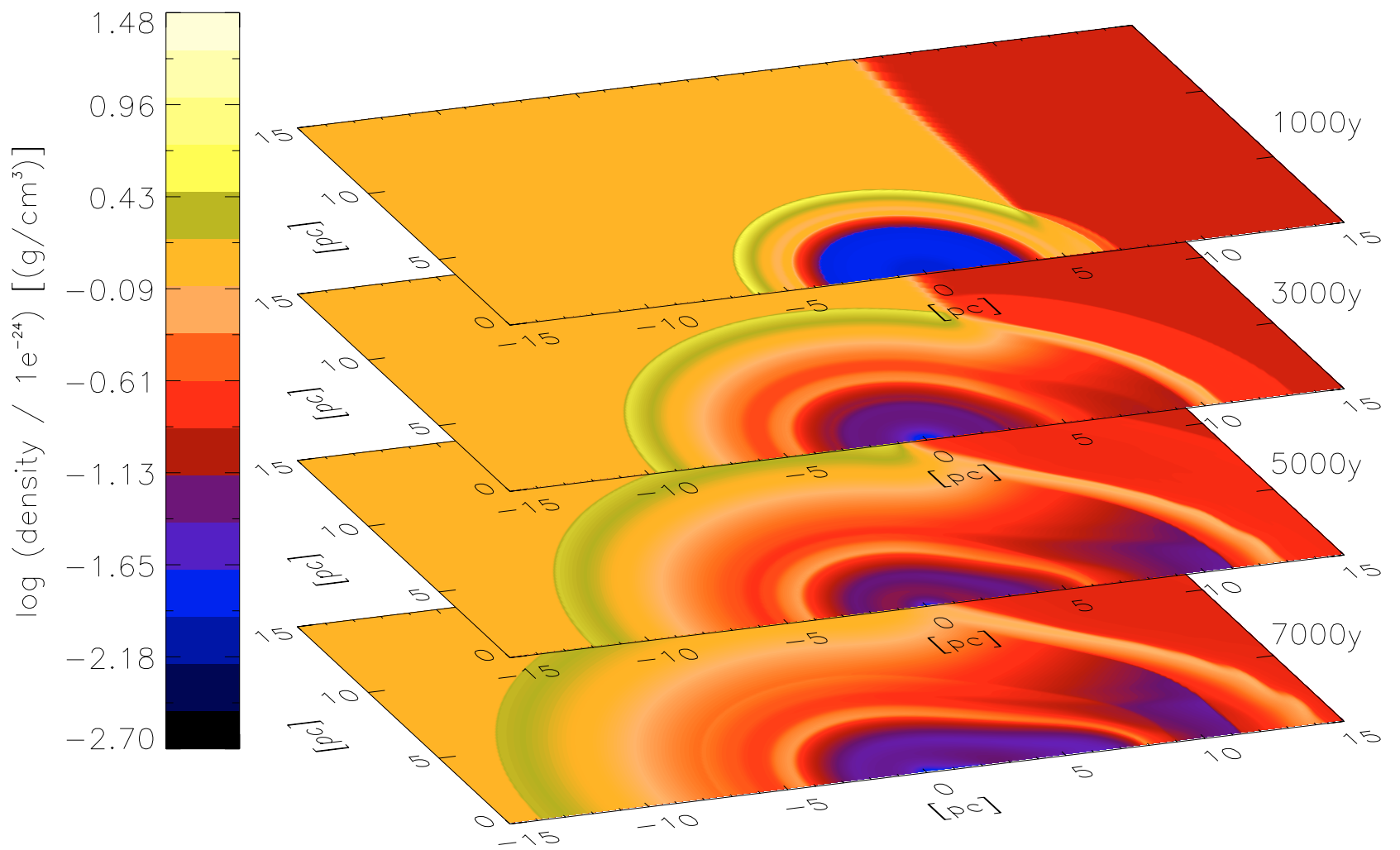

Fig. 8. Similar to Fig. 5, except here the SNR moves from a medium with density of $\rho_{\mathrm{ISM}}=10^{-24} \mathrm{~g} \mathrm{~cm}^{-3}$ to a medium where $\rho_{\mathrm{ISM}}=10^{-25} \mathrm{~g} \mathrm{~cm}{ }^{-3}$. In this case densities of $\rho_{\mathrm{ISM}}=10^{-24} \mathrm{~g} \mathrm{~cm}^{-3}$ will be the denser region. 

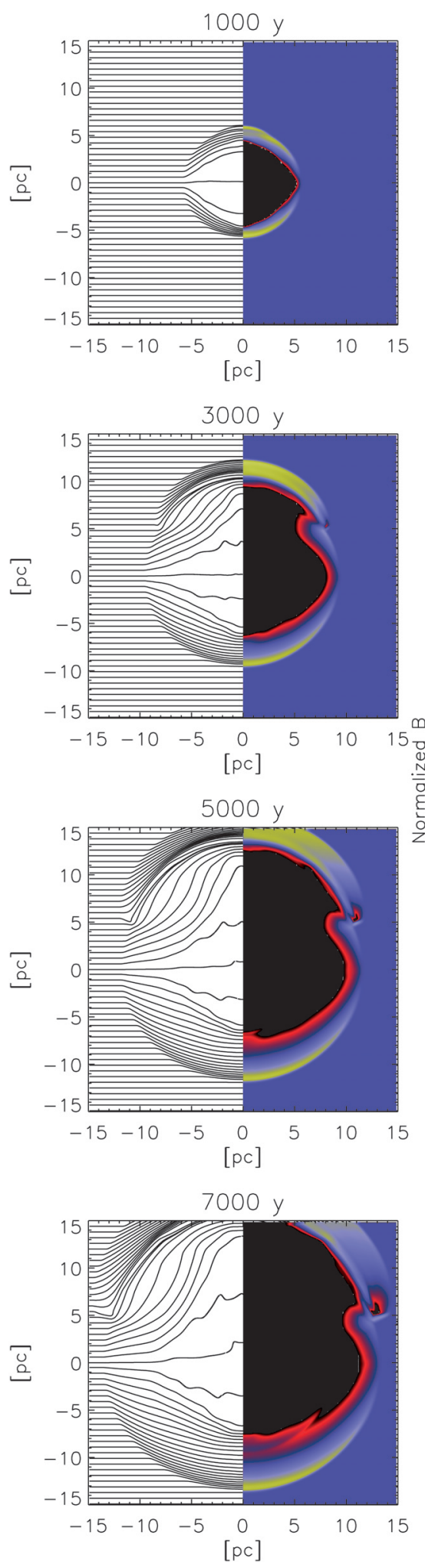

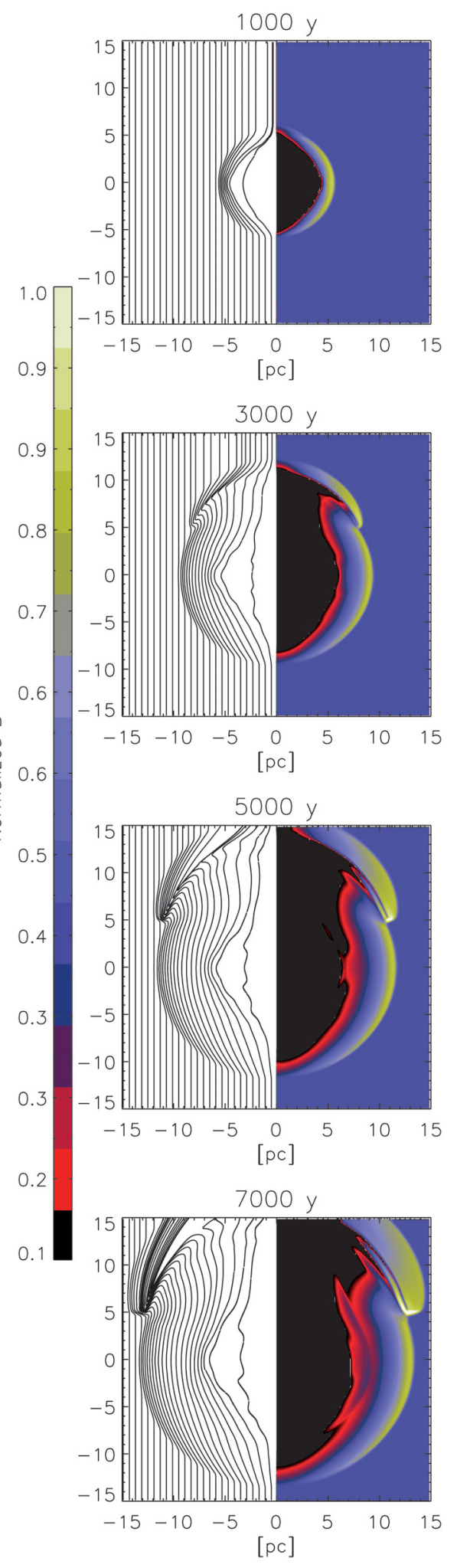

Fig. 9. As in Fig. 6 but for the case where the SNR moves into a less denser region.

dotted lines show the parameters directly away on the opposite side.

Most interesting here is the absence of a reflected wave as in the previous scenario, also the RS (with a rarefraction region behind) will reach the center at the same time irrespective of its position. However, it is also interesting that once the SNR FS moves into the medium of lower density, a second RS will start to evolve in this region. This is evident on the bottom right panel where a large pressure gradient is starting to form. After the first RS have reached the center, this second RS will start to move inward.

This effect is better illustrated in Fig. 11 showing the pressure $P$ of the SNR in the direction directly toward the contact discontinuity. The simulation is shown from $2000 \mathrm{yr}$ up to $8000 \mathrm{yr}$. 


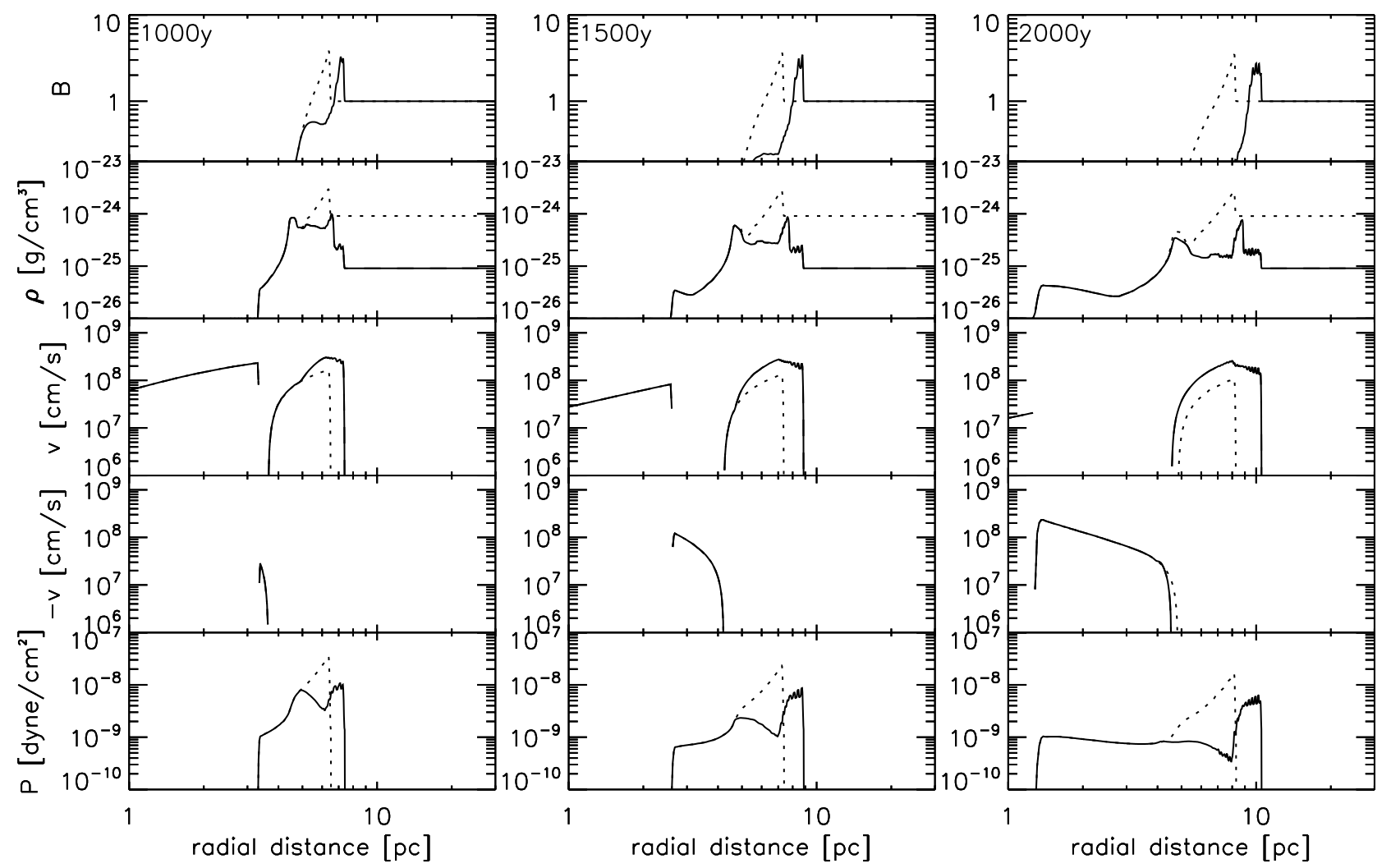

Fig. 10. As in Fig. 7 except here the SNR moves into a medium of lesser density. The interface between the two media is a infinite plane situated $5 \mathrm{pc}$ from the initial explosion. Note that in this scenario there is no reflected shock, but a second reverse shock start to form as the FS moves well into the medium of lesser density.

Shown here, is that as time increases the second RS becomes more pronounced. In particular at $4000 \mathrm{yr}$ it is clearly visible, and also a contact discontinuity between the swept-up material and original ejecta. However at this time the original RS have already reached the center. As time increases the second RS start to became stationary and at $8000 \mathrm{yr}$ it starts to move toward the center.

\section{Examples}

In this section some examples are discussed which can be compared to the computations shown above.

\subsection{The Vela SNR}

ROSAT observations of the thermal X-ray component of the Vela SNR have revealed an approximate SNR diameter of 8 degrees, which translates to a diameter of $40 \mathrm{pc}$ for a pulsar distance of 290 pc. Blondin et al. (2001) already remarked that the ISM around Vela is inhomogeneous: these authors summarized $\mathrm{X}$-ray and HI observations, revealing densities between 0.06 and $1 \mathrm{~cm}^{-3}$, with the latter mostly confined to the northern region where bright thermal X-rays are seen in the ROSAT image. From Fig. 2 we deduce a forward shock radius $R_{1} \sim 17$ pc (the distance from the projected Vela birth place to the northern edge of the SNR) for a number density of $n=1 \mathrm{~cm}^{-3}$ (or mass density of $10^{-24} \mathrm{~g} \mathrm{~cm}^{-3}$ after $t_{\mathrm{SNR}} \sim 11000 \mathrm{yr}$, which is consistent with the preshock density to the north derived from HI observations (Dubner et al. 1998). For these parameters we see that the reverse shock from the north already returned to the center of the SNR after $4000 \mathrm{yr}$.
The largest radius of the Vela SNR $\left(R_{1} \sim 23 \mathrm{pc}\right.$ from the pulsar) is seen towards the southwestern section, where the preshock density was probably also lowest (i.e. lowest thermal $\mathrm{X}$-ray surface brightness). For an average preshock density of $n \sim 0.1 \mathrm{~cm}^{-3}$, we find that the predicted forward shock radius is indeed $\sim 23$ pc after 11000 yr. For this lower density, the reverse shock would have returned after $\sim 8000 \mathrm{yr}$, but since the reverse shock from the northern part already arrived after $4000 \mathrm{yr}$ at the center, this northern reverse shock would have swept the much later arriving southwestern reverse shock back towards its origin.

The net result is that we observe the radio PWN Vela X (with an extension of $3^{\circ} \times 2^{\circ}$ ) to be offset to the south of the pulsar, whereas the X-ray cocoon is offset to the south-southwest of the pulsar (Markwardt \& Ogelman 1997). The age of the cocoon is quite important in discussions of leptonic versus hadronic interpretation (de Jager 2007b,a) of the HESS signal as seen by Aharonian et al. (2006a). Since the cocoon must have been offset from the pulsar a few thousand years ago, electrons in the southern tip of the offset cocoon emitting synchrotron X-rays with energy $E_{\mathrm{X}} \mathrm{keV}$ in a field of strength $B$ would survive up to an age of $\tau=1.2 \mathrm{kyr}(B / 10 \mu \mathrm{G})^{-3 / 2}\left(E_{\mathrm{X}} / 1 \mathrm{keV}\right)^{-1 / 2}$ (de Jager 2007a). Here $\tau=t_{\mathrm{SNR}}-t_{\mathrm{R}}$ is the time since the reverse shock shifted the synchrotron emitting electrons to the south, while $t_{\mathrm{R}}$ is the time since birth for the PWN offset to be completed, as discussed above. The field strength must be low enough for 10 $\mathrm{keV}$ synchrotron emitting electrons to survive in the cocoon: by assuming $t_{\mathrm{R}}=7000 \mathrm{yr}$, which consists of the $4000 \mathrm{yr}$ for the reverse shock to reach the center as discussed above, plus approximately another $3000 \mathrm{yr}$ for the offset towards the south to be completed, with the latter number derived from the simulations of Blondin et al. (2001), we arrive at $\tau \sim 4000 \mathrm{yr}$ for the synchrotron lifetime of these electrons in the southern tip of the 


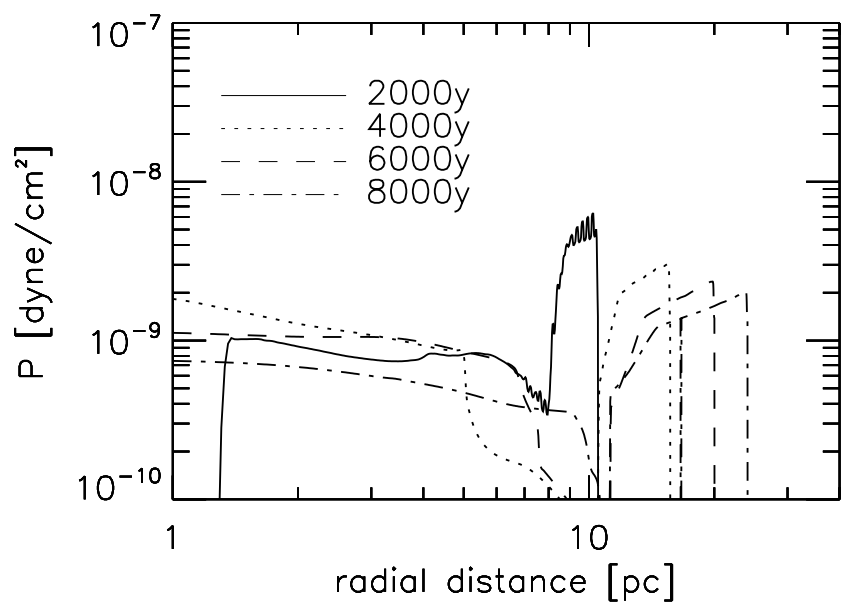

Fig. 11. The pressure $P$ of the SNR in the direction toward the contact discontinuity. The simulation is shown from $2000 \mathrm{yr}$ up to $8000 \mathrm{yr}$. As time increases a second RS forms and at $8000 \mathrm{yr}$ it starts to move toward the center.

X-ray cocoon. This implies a cocoon field strength of $B \sim 2 \mu \mathrm{G}$, which is remarkably close to the lower limit for $B$ as required by a leptonic interpretation. de Jager (2007a) also showed that subBohm cross field diffusion is expected for a cocoon wrapped in a toroidal field structure, which would allow the cocoon to contain the synchrotron emitting electons to the present day (note that further cocoon expansion under its own pressure results in magnetic induction and hence toroidal field structures around the cocoon).

Note that we did not include the pressure of the expanding PWN. Inclusion of this effect would have reduced the slope of the plot of reverse shock radius vs. time significantly, since the time it takes for the reverse shock to offset the PWN may take a few kyr to complete as discussed above (Blondin et al. 2001). Therefore, if the pressure of the expanding PWN were to be included, the time for the reverse shock to return to the origin (and offset the PWN), would be larger than indicated in the bottom panel of Fig. 2.

The next step would be to redo these simulations after inclusion of the expanding PWN, which would modify $t_{\mathrm{R}}$ (as discussed above) to give eventually more accurate numbers for the cocoon field strength.

\subsection{HESS J1825-17=G18.0-0.7}

The Vela-like pulsar PSR B1823-13, with a spindown age of $21000 \mathrm{yr}$, is the source of the X-ray PWN G18.0-0.7, which shows a source extension of 5 arcmin (Gaensler et al. 2003) whereas very high energy gamma-ray emission with a much larger source extent was seen with the HESS telescopes (Aharonian et al. 2006b). The gamma-ray emission of the source HESS J1825-137 is clearly offset to the south-west of the pulsar. The leptonic origin of the gamma-ray signal is demonstrated by the energy dependence of the morphology: the highest energy emission is located close to the pulsar, whereas the lower energy emission extends about 1 degree away from the pulsar (toward the south-west), which can be explained in terms of energy dependent synchrotron cooling. This one-sidedness, similar to Vela, can also be explained in terms of a reverse shock, which shifted the PWN HESS J1825-137 also towards the south-west (Aharonian et al. 2006b). The dimensions of this PWN is 0.6 by 1 degree, with the latter's additional extension probably caused by the smearing of the reverse shock. We will therefore take the intrinsic diameter to be 0.6 degrees.

A source radius of 0.3 degrees translates to a PWN radius of $20 \mathrm{pc}$, given the pulsar based dispersion measure of $3.9 \mathrm{kpc}$. From Table 1 of composite SNR considered by van der Swaluw $\& \mathrm{Wu}$ (2001), we see that the forward shock radii $R_{1}$ of all remnants are at least four times larger than the PWN radius. This would imply a FS radius of $R_{1}=80 \mathrm{pc}$ towards the south-west where the extension is the largest. From Fig. 2 we see that this radius is achieved after $21000 \mathrm{yr}$ for a density of $6 \times 10^{-4} \mathrm{~cm}^{-3}$, which corresponds to the lowest densities expected for the hot phase of the ISM (e.g. Ferriere 1998, with references therein). Such low densities would be possible if a bubble was blown by the stellar winds of massive stars born in or near molecular clouds. In fact, Lemiere et al. (2005) found evidence for a molecular cloud at the same kinematic distance as PSR B182313 , but located such that (a) young massive stars formed in the cloud could have blown the bubble towards the south-west, while ablating part of the cloud; (b) a combination reverse shock and reflection wave resulted in the offset PWN of the SNR of PSR B1823-13 towards the south-west, as observed.

The next step would be to add the evolving PWN to these simulations to see if the observed VHE gamma-ray morphology can constrain the SNR/ISM parameters. The first results which are discussed by (de Jager \& Ferreira 2007) show the effect of the pulsar spindown timescale on the evolving morphology of this source.

\subsection{A reflection wave in $\mathrm{G} 21.5-0.9$ ?}

Even though a radio pulsar (PSR J1833-1034) was finally discovered in the plerion of the composite remnant G21.5-0.9, its spindown age of $4.8 \mathrm{kyr}$ appears to be inconsistent with the properties of the associated SNR (Camilo et al. 2006). The shell appears to be still in the free expanding phase and an age less than 1000 yr was derived by these authors.

Investigation of the halo subtracted image of this composite remnant (Fig. 9 of Bocchino et al. 2005) shows that the remnant is not symmetric relative to the pulsar position. The extension to the north is smaller than the corresponding extension to the south, which indicates that the ISM is denser towards the north, where the most dominant feature is the "North Spur", showing bright nonthermal and thermal emission. This feature, together with a bright ridge, is located just inside the northern SNR rim (or forward shock location).

Since the synchrotron emission in the North Spur (and associated ridge) is brighter than the corresponding emission in the southern part of the SNR, we invoke a situation, as sketched in Sect. 4, where the northern forward shock struck a wall of denser material (e.g. a molecular cloud), which resulted in the relatively smaller forward shock radius to the north. A reflected wave, originating near the forward shock radius, is now returning to the origin of the SNR explosion and since it is still in its early phase (located at the North Spur), it still carries significant magnetic azimuthally directed magnetic flux towards the south, as shown in the left panels of Fig. 6. Furthermore, from this figure we see that the magnetic field strength in the reflected wave is larger in the northern half of the SNR compared to the southern mirror points, and the same wave is also expected to carry Fermi accelerated electrons with it. The reason for this is the azimuthal structure of the field in the reflected wave. We therefore get synchrotron emission from both the forward shock as well as from the reflected wave, giving the appearance of a radially broadened ridge of which the brightest part is the North Spur. 
Furthermore, since the gas density towards the north is also greater compared to the south (see Fig. 7), the thermal emission near the North Spur is also expected to be brighter compared to the southern section of the SNR.

\section{Summary and conclusions}

In this work numerical simulations showing the time evolution of a SNR in uniform and non-uniform media were presented. For this we used a hydrodynamic model solving the well known Euler equations. Different from previous studies is that we also include a kinematic calculation (solving the induction equation) of the interstellar magnetic field (LeVeque 2002; Fahr et al. 2000; Scherer \& Ferreira 2005; Ferreira \& Scherer 2006).

We started by showing how various parameters, such as the ejecta mass $M_{\text {ej }}$, energy $E_{\text {ej }}$, as well as ISM density $\rho_{\text {ISM }}$ and adiabatic index $\gamma$ influence the evolution of a SNR in a uniform medium. In particular we were interested calculating the return time $t_{\mathrm{R}}$ for when the RS has reached the origin of the explosion. By varying the different parameters we found that

$-t_{\mathrm{R}} \propto \rho_{\mathrm{ISM}}^{-1 / 3}$

$-t_{\mathrm{R}} \propto M_{\mathrm{ej}}^{3 / 4}$

$-t_{\mathrm{R}} \propto E_{\mathrm{ej}}^{-45 / 100}$

$-t_{\mathrm{R}} \propto \gamma^{-3 / 2}$.

Variations in the last parameter are expected due to the nonlinear interaction of accelerated cosmic rays on SNR shocks (e.g. Decourchelle et al. 2000; Ellison et al. 2004). These were all combined in Eq. (16) giving an analytical expression for $t_{\mathrm{R}}$ as a function of all the parameters above. Note that in this work we neglect the existence of a PWN inside a SNR in our calculations.

For the second part we concentrated on SNR evolution in non-uniform media (see also Voelk 1983; Tenorio-Tagle et al. 1991; Borkowski et al. 1997; Dohm-Palmer \& Jones 1996; Jun \& Jones 1999). The simple scenario, where a SNR moves from one medium into either a less or more dense medium, is studied. For simplicity we assumed that the interface between these two media is an infinite plane situated $5 \mathrm{pc}$ from the initial explosion. It was shown how varoius parameters, such as density, speed, pressure and the compressed ISM magnetic field changes when a SNR moves into another medium with a different density (e.g. from the ISM into a molecular could or vice versa).

As the SNR moves into another medium of higher density the following is computed:

- The blast wave, FS, decelerates because of the increase in mass (momentum conservation). This results in the SNR geometry deviating significantly from a sphere as time increases.

- A reflection wave is created which is driven back toward the center. This drives mass nonspherically away from the discontinuity which is no longer exhibiting its planar geometry.

- As the reflection shock moves inward it drags some of the ISM field lines with it (only when the field is parallel to the interface). The result is that the field inside the SNR next to the medium of higher density are now considerably larger compared to the opposite direction.

- The reflected wave catches up with the RS. As this wave propagates inward it heats the inside of the SNR resulting in larger temperatures in this region compared to the other side.

We also concentrated on a scenario where the opposite occurs. A SNR explodes in a medium with a high density and the blast waves then propagates into a medium with a lower density. The following is of interest:

- As the blast wave inside a dense medium moves into a less dense medium a cavity is being blown away changing the interface between the two media.

- Once the SNR moves into the medium of less density, a second RS will start to evolve in this region. With the parameters chosen in this work our calculations show that after the first $\mathrm{RS}$ have reached the center, this second RS will also start to move inward.

Lastly three observed sources were discussed and compared to simulations presented in this work. We showed that

- When assuming the surrounding ISM around Vela is inhomogeneous our model predicts a FS radius of $\sim 17 \mathrm{pc}$ after $11000 \mathrm{yr}$ to the northern edge assuming a number density of $n=1 \mathrm{~cm}^{-3}$ for the ISM. For the southwest region the largest radius is observed and for an average density of $n=\sim 0.1 \mathrm{~cm}^{-3}$ we find the FS radius to be $\sim 23 \mathrm{pc}$ in this region.

- A reverse shock/reflection wave combination of the SNR of PSR B1823-13 could result in the offset PWN towards the south-west, as observed. This would imply a maximal radius of $R_{1}=80 \mathrm{pc}$ for the FS towards the south-west where the extension is the largest. From Fig. 2 we see that this radius is achieved after $21000 \mathrm{yr}$ for a density of $6 \times 10^{-4} \mathrm{~cm}^{-3}$.

- The remnant G21.5-0.9 is not symmetric relative to its pulsar position: the extension to the north is smaller than the corresponding extension to the south, which indicates that the ISM is denser towards the north, where the most dominant feature is the "North Spur" showing bright nonthermal and thermal emission. Since the synchrotron emission in the North Spur is brighter than in the southern part of the SNR, we invoke a situation, as sketched in Sect. 4, where the northern FS struck a wall of denser material (e.g. a molecular cloud), which resulted in the relatively smaller FS radius to the north. A reflected wave, originating near the FS radius, is now returning to the origin of the SNR explosion and since it is still in its early phase (located at the North Spur), it still carries significant magnetic azimuthally directed magnetic flux towards the south, as was shown in Fig. 6. Furthermore, from Fig. 6 we saw that the magnetic field strength in the reflected wave is larger in the northern half of the SNR compared to the southern mirror points, and the same wave is also expected to carry Fermi accelerated electrons with it. The reason for this is the azimuthal structure of the field in the reflected wave. We therefore get synchrotron emission from both the FS as well as from the reflected wave, giving the appearance of a radially broadened ridge of which the brightest part is the North Spur.

Acknowledgements. The authors are grateful for partial financial support granted to them by the South African National Research Foundation (NRF) and by the Meraka Institute as part of the funding for the South African Centre for High Performance Computing (CHPC).

\section{References}

Aharonian, F., Akhperjanian, A. G., Bazer-Bachi, A. R., et al. 2006a, A\&A, 448, L43

Aharonian, F., Akhperjanian, A. G., Bazer-Bachi, A. R., et al. 2006b, A\&A, 460, 365

Ardavan, H. 1973, ApJ, 184, 435

Blondin, J. M., \& Ellison, D. C. 2001, ApJ, 560, 244 
Blondin, J. M., Chevalier, R. A., \& Frierson, D. M. 2001, ApJ, 563, 806 Bocchino, F., van der Swaluw, E., Chevalier, R., \& Bandiera, R. 2005, A\&A, 442,539

Borkowski, K. J., Blondin, J. M., \& McCray, R. 1997, ApJ, 477, 281

Bucciantini, N., Blondin, J. M., Del Zanna, L., \& Amato, E. 2003, A\&A, 405, 617

Bucciantini, N., Amato, E., Bandiera, R., Blondin, J. M., \& Del Zanna, L. 2004, A\&A, 423, 253

Camilo, F., Ransom, S. M., Gaensler, B. M., et al. 2006, ApJ, 637, 456

Cioffi, D. F., McKee, C. F., \& Bertschinger, E. 1988, ApJ, 334, 252

de Jager, O. C. 2007a, Lecture notes in Physics, in press

de Jager, O. C. 2007b, ApJ, 658, 1177

de Jager, O. C., \& Ferreira, S. E. S. 2007, Ap\&SS, submitted

Decourchelle, A., Ellison, D. C., \& Ballet, J. 2000, ApJ, 543, L57

Del Zanna, L., Amato, E., \& Bucciantini, N. 2004, A\&A, 421, 1063

Dohm-Palmer, R. C., \& Jones, T. W. 1996, ApJ, 471, 279

Dubner, G. M., Green, A. J., Goss, W. M., Bock, D. C.-J., \& Giacani, E. 1998 AJ, 116, 813

Dwarkadas, V. V., \& Chevalier, R. A. 1998, ApJ, 497, 807

Ellison, D. C., Decourchelle, A., \& Ballet, J. 2004, A\&A, 413, 189

Fahr, H. J., Kausch, T., \& Scherer, H. 2000, A\&A, 357, 268

Ferreira, S. E. S., \& Scherer, K. 2006, ApJ, 642, 1256

Ferriere, K. 1998, ApJ, 503, 700
Gaensler, B. M., Schulz, N. S., Kaspi, V. M., Pivovaroff, M. J., \& Becker, W. E. 2003, ApJ, 588, 441

Jun, B.-I., \& Jones, T. W. 1999, ApJ, 511, 774

Lemiere, A., Terrier, R., \& Djannati, A. 2005, in SF2A-2005: Semaine de 1'Astrophysique Francaise, ed. F. Casoli, T. Contini, J. M. Hameury, \& L. Pagani, 479

LeVeque, R. J. 2002, Finite Volume Methods for Hyperbolic Problems (Cambridge University Press)

Markwardt, C. B., \& Ogelman, H. B. 1997, ApJ, 480, L13

McKee, C. F. 1974, ApJ, 188, 335

McKee, C. F., \& Truelove, J. K. 1995, Phys. Rep., 256, 157

Scherer, K., \& Ferreira, S. E. S. 2005, Astrophys. Space Sci. Trans., 1, 17

Sedov, L. I. 1959, Similarity and Dimensional Methods in Mechanics, Similarity and Dimensional Methods in Mechanics (New York: Academic Press)

Tang, S., \& Wang, Q. D. 2005, ApJ, 628, 205

Tenorio-Tagle, G., Rozyczka, M., Franco, J., \& Bodenheimer, P. 1991, MNRAS, 251,318

Truelove, J. K., \& McKee, C. F. 1999, ApJS, 120, 299

van der Swaluw, E., \& Wu, Y. 2001, ApJ, 555, L49

van der Swaluw, E., Achterberg, A., Gallant, Y. A., \& Tóth, G. 2001, A\&A, 380, 309

Voelk, H. J. 1983, Space Sci. Rev., 36, 3

Woltjer, L. 1972, ARA\&A, 10, 129 